\title{
THE
}

$9-1998$

\section{Static and Dynamic Structure Factors in the Haldane Phase of the Bilinear-Biquadratic Spin-1 Chain}

Andreas Schmitt

Karl-Heinz Mutter

Michael Karbach

University of Rhode Island

Yongmin $\mathrm{Yu}$

University of Rhode Island

Gerhard Müller

University of Rhode Island, gmuller@uri.edu

Follow this and additional works at: https://digitalcommons.uri.edu/phys_facpubs

Terms of Use

All rights reserved under copyright.

\section{Citation/Publisher Attribution}

Schmitt, A., Mütter, K.-H., Karbach, M., Yu, Y., \& Müller, G. (1998). Static and dynamic structure factors in the Haldane phase of the bilinear-biquadratic spin-1 chain. Physical Review B, 58(9), 5498-5508. doi: 10.1103/PhysRevB.58.5498

Available at: http://dx.doi.org/10.1103/PhysRevB.58.5498

This Article is brought to you for free and open access by the Physics at DigitalCommons@URI. It has been accepted for inclusion in Physics Faculty Publications by an authorized administrator of DigitalCommons@URI. For more information, please contact digitalcommons-group@uri.edu. 


\title{
Static and dynamic structure factors in the Haldane phase of the bilinear-biquadratic spin-1 chain
}

\author{
Andreas Schmitt and Karl-Heinz Mütter \\ Department of Physics, University of Wuppertal, D-42097 Wuppertal, Germany \\ Michael Karbach,* Yongmin Yu, and Gerhard Müller \\ Department of Physics, The University of Rhode Island, Kingston, Rhode Island 02881-0817
}

(Received 11 March 1998)

\begin{abstract}
The excitation spectra of the $T=0$ dynamic structure factors for the spin, dimer, and trimer fluctuation operators as well as for the center fluctuation operator in the one-dimensional $S=1$ Heisenberg model with isotropic bilinear $(J \cos \theta)$ and biquadratic $(J \sin \theta)$ exchange are investigated via the recursion method for systems with up to $N=18$ sites over the predicted range, $-\pi / 4<\theta \leq \pi / 4$, of the topologically ordered Haldane phase. The four static and dynamic structure factors probe the ordering tendencies in the various coupling regimes and the elementary and composite excitations which dominate the $T=0$ dynamics. At $\theta=\arctan \frac{1}{3}$ (valence-bond solid point), the dynamically relevant spectra in the invariant subspaces with total spin $S_{T}$ $=0,1,2$ are dominated by a branch of magnon states $\left(S_{T}=1\right)$, by continua of two-magnon scattering states $\left(S_{T}=0,1,2\right)$, and by discrete branches of two-magnon bound states with positive interaction energy $\left(S_{T}\right.$ $=0,2)$. The dimer and trimer spectra at $q=\pi$ are found to consist of single modes with $N$-independent excitation energies $\omega_{\lambda}^{D} /\left|e_{0}\right|=5$ and $\omega_{\lambda}^{T} /\left|e_{0}\right|=6$, where $e_{0}=E_{0} / N$ is the ground-state energy per site. The basic structure of the dynamically relevant excitation spectrum remains the same over a substantial parameter range within the Haldane phase. At the transition to the dimerized phase $(\theta=-\pi / 4)$, the two-magnon excitations turn into two-spinon excitations. [S0163-1829(98)00233-1]
\end{abstract}

\section{INTRODUCTION}

The zero-temperature phase diagram of the onedimensional (1D) $S=1$ Heisenberg model with bilinear and biquadratic exchange,

$$
H_{\theta}=J \sum_{l=1}^{N}\left[\cos \theta\left(\mathbf{S}_{l} \cdot \mathbf{S}_{l+1}\right)+\sin \theta\left(\mathbf{S}_{l} \cdot \mathbf{S}_{l+1}\right)^{2}\right]
$$

$-\pi<\theta \leqslant \pi$, as is widely accepted today, ${ }^{1-4}$ consists of a phase with ferromagnetic long-range order $(\theta<-3 \pi / 4$ or $\theta>\pi / 2)$, a phase with dimer long-range order $(-3 \pi / 4<\theta$ $<-\pi / 4)$, the Haldane phase with hidden topological longrange order $(-\pi / 4<\theta<\pi / 4)$, and a somewhat obscure "trimerized" phase $(\pi / 4<\theta<\pi / 2) . H_{\theta}$ is Bethe ansatz solvable at $\theta=-\pi / 4,{ }^{5}$ and at $\theta=\pi / 4,{ }^{6}$ and for part of the eigenvalue spectrum also at $\theta=-\pi / 2 .^{7}$ Within the Haldane phase, at the parameter value $\theta_{\mathrm{VBS}}=\arctan \frac{1}{3} \simeq 0.1024 \pi$, the groundstate wave function of $H_{\theta}$ is a realization of the (explicitly known) valence-bond solid (VBS) wave function. ${ }^{8-10}$

In spite of numerous theoretical and computational studies of this model system, there are still many blank spots on the map, especially with respect to dynamical properties. A panoramic view of the various ordering tendencies and of the dominant quantum fluctuations at $T=0$ can be gained from a study of the dynamic structure factors,

$$
S_{A A}(q, \omega) \equiv \int_{-\infty}^{+\infty} d t e^{i \omega t}\left\langle F_{q}^{A}(t) F_{q}^{A^{\dagger}}\right\rangle
$$

for a set of fluctuation operators $F_{q}^{A}$. Among them should be the operators which, for specific wave numbers $q$, describe the known or suspected order parameters. Further fluctuation operators may be chosen according to specific symmetry properties of $H_{\theta}$, which entail special selection rules.

Each dynamic structure factor calculated for the same parameter value $\theta$ has its own dynamically relevant excitation spectrum and its own spectral-weight distribution. Each fluctuation operator acts like a filter on the complete eigenvalue spectrum, and the associated dynamic structure factor highlights a particular subset of excitations. Looking at the $T$ $=0$ dynamics of $H_{\theta}$ through a series of such filters reveals many interesting features that are not readily accessible by any other means. The composite structure of parts of the excitation spectrum and the nature of the underlying elementary excitations, for example, may be recognized only if observed through the right set of filters.

For some questions, it is useful to investigate the corresponding static structure factors,

$$
S_{A A}(q) \equiv\left\langle F_{q}^{A} F_{q}^{A^{\dagger}}\right\rangle=\int_{-\infty}^{+\infty} \frac{d \omega}{2 \pi} S_{A A}(q, \omega),
$$

which are more likely amenable to a finite-size scaling analysis. All these quantities can be computed from the finite-size ground-state wave function, $S_{A A}(q)$ directly as an expectation value and $S_{A A}(q, \omega)$ indirectly via the recursion method. ${ }^{11,12}$

The fluctuation operators used in this study and their relation to different order parameters are introduced in Sec. II. The static and dynamic structure factors which probe the 
different kinds of fluctuations are investigated in Sec. III for the VBS point and in Sec. IV for the surrounding parts of the Haldane phase. The continuation of this study for the parameter values at the two critical points $\theta= \pm \pi / 4$ and in the phases beyond these points will be reported elsewhere. ${ }^{13}$

\section{FLUCTUATION OPERATORS AND ORDER PARAMETERS}

For the study of the model system (1.1) we introduce four different fluctuation operators of the general form

$$
F_{q}^{A} \equiv \frac{1}{\sqrt{N}} \sum_{l=1}^{N} e^{i q l} A_{l},
$$

where the operator $A_{l}$ acts locally at lattice site $l$ and (in some cases) also on one or two neighboring sites.

(i) The spin fluctuations are probed by the operator $F_{q}^{S}$ with

$$
S_{l} \equiv S_{l}^{3}=\left(\begin{array}{ccc}
1 & 0 & 0 \\
0 & 0 & 0 \\
0 & 0 & -1
\end{array}\right) \text {. }
$$

For $q=0$ they represent ferromagnetic order-parameter fluctuations and for $q=\pi$ Néel order-parameter fluctuations. Ferromagnetic long-range order does exist in this model, but Néel long-range order does not. The Néel order-parameter fluctuations are expected to be strongest at the critical point $\theta=-\pi / 4$, which marks the disappearance of topological long-range order, the only point in the phase diagram where the $q=\pi$ spin excitations are known to be gapless.

(ii) The dimer fluctuations are characterized by the operator $F_{q}^{D}$ with $^{14}$

$$
D_{l} \equiv \mathbf{S}_{l} \cdot \mathbf{S}_{l+1}-\left\langle\mathbf{S}_{l} \cdot \mathbf{S}_{l+1}\right\rangle .
$$

The dimer order-parameter fluctuations, probed by $F_{\pi}^{D}$, are expected to be strongest at the same critical point, $\theta$ $=-\pi / 4$, which also marks the onset of dimer long-range order.

(iii) For the study of trimer fluctuations, we use the operator $F_{q}^{T}$ with

$$
T_{l} \equiv P_{l}^{T}-\left\langle P_{l}^{T}\right\rangle
$$

constructed from projection operators

$$
P_{l}^{T} \equiv|[l, l+1, l+2]\rangle\langle[l, l+1, l+2]|
$$

onto local trimer states

$$
\begin{aligned}
|[1,2,3]\rangle \equiv & \frac{1}{\sqrt{6}}(|+0-\rangle+|0-+\rangle+|-+0\rangle \\
& -|-0+\rangle-|0+-\rangle-|+-0\rangle),
\end{aligned}
$$

which are completely antisymmetric states with total spin $S_{T}=0 .{ }^{15}$ The state (2.6) is, in fact, the (nondegenerate) ground state of $H_{\theta}$ with $N=3$ for $\arctan \frac{1}{3} \leqslant \theta \leqslant \pi / 2$. This observation was interpreted as suggesting that a phase with trimer long-range order might exist for $N \rightarrow \infty$. The trimer order-parameter fluctuations are probed by $F_{2 \pi / 3}^{T}$. (iv) Our finite- $N$ data indicate that in the same parameter range, $\pi / 4 \leqslant \theta \leqslant \pi / 2$, where the trimer order-parameter fluctuations are strong, the spin fluctuations are significantly stronger, and the fluctuations of a modified spin operator, which tunes into existing period-three patterns of local upzero-down $(+, 0,-)$ or down-zero-up $(-, 0,+)$ spin states are even stronger. The center fluctuation operator $F_{q}^{Z}$ is constructed from the matrices

$$
Z_{l} \equiv Z_{l}^{3}=\left(\begin{array}{ccc}
e^{i 2 \pi / 3} & 0 & 0 \\
0 & 1 & 0 \\
0 & 0 & e^{-i 2 \pi / 3}
\end{array}\right)=\mathbf{1}+i \frac{\sqrt{3}}{2} S_{l}^{z}-\frac{3}{2}\left(S_{l}^{z}\right)^{2},
$$

which is an element of the $\mathrm{SU}(3)$ center. ${ }^{16}$ The center order parameter has the same wave number, $q=2 \pi / 3$, as the trimer order parameter. At $\theta=\pi / 4$ the excitation spectrum of $H_{\theta}$ is rigorously known to be gapless for this wave number.

In the Appendix we discuss the static spin, dimer, trimer, and center correlation functions in special states constructed such as to reflect pure Néel, dimer, trimer, or center longrange order.

All four fluctuation operators $F_{q}^{A}$ are invariant under (discrete) translations in real space and under (continuous) rotations about the 3 axis in spin space. The resulting selection rules for the excited states that may contribute to any of the four dynamic structure factors $S_{A A}(q, \omega)$ are $\Delta k=q$ for the wave number and $\Delta S_{T}^{3}=0$ for the 3 component of the total spin.

Only two of the fluctuation operators are fully rotationally invariant, $\left[F_{q}^{D}, S_{T}^{i}\right]=\left[F_{q}^{T}, S_{T}^{i}\right]=0$ for $i=1,2,3$ and arbitrary $q$. This produces the additional selection rules $\Delta S_{T}=0$ for the magnitude of the total spin in the dynamic dimer and trimer structure factors. The corresponding selection rules for the dynamic spin and center structure factors are $\Delta S_{T}=0,1$ and $\Delta S_{T}=0,1,2$, respectively, with the further restriction that transitions between singlets $\left(S_{T}=0\right.$ states $)$ are prohibited.

In the nonferromagnetic parameter range $(-3 \pi / 4<\theta$ $<\pi / 2$ ), where the finite- $N$ ground state is a nondegenerate singlet, the dynamic structure factors $S_{D D}(q, \omega)$ and $S_{T T}(q, \omega)$ thus couple exclusively to the $S_{T}=0$ excitation spectrum, and $S_{S S}(q, \omega)$ exclusively to the $S_{T}=1$ excitation spectrum, whereas $S_{Z Z}(q, \omega)$ couples to the $S_{T}=1$ and $S_{T}$ $=2$ spectra.

To calculate $S_{A A}(q, \omega)$ we employ the recursion $\operatorname{method}^{17,11}$ in combination with a finite-size continuedfraction analysis. ${ }^{12}$ The recursion algorithm in the present context is based on an orthogonal expansion of the wave function $\left|\Psi_{q}^{A}(t)\right\rangle=F_{q}^{A}(-t)|G\rangle$, where $|G\rangle$ is the finite- $N$ ground state. It produces a double sequence of continuedfraction coefficients $\left\{a_{k}(q), b_{k}^{2}(q)\right\}$ for the function

$$
d_{0}^{A A}(q, \zeta)=\frac{i}{\zeta-a_{0}(q)-\frac{b_{1}^{2}(q)}{\zeta-a_{1}(q)-\frac{b_{2}^{2}(q)}{\zeta-a_{2}(q)-\ldots}}},
$$



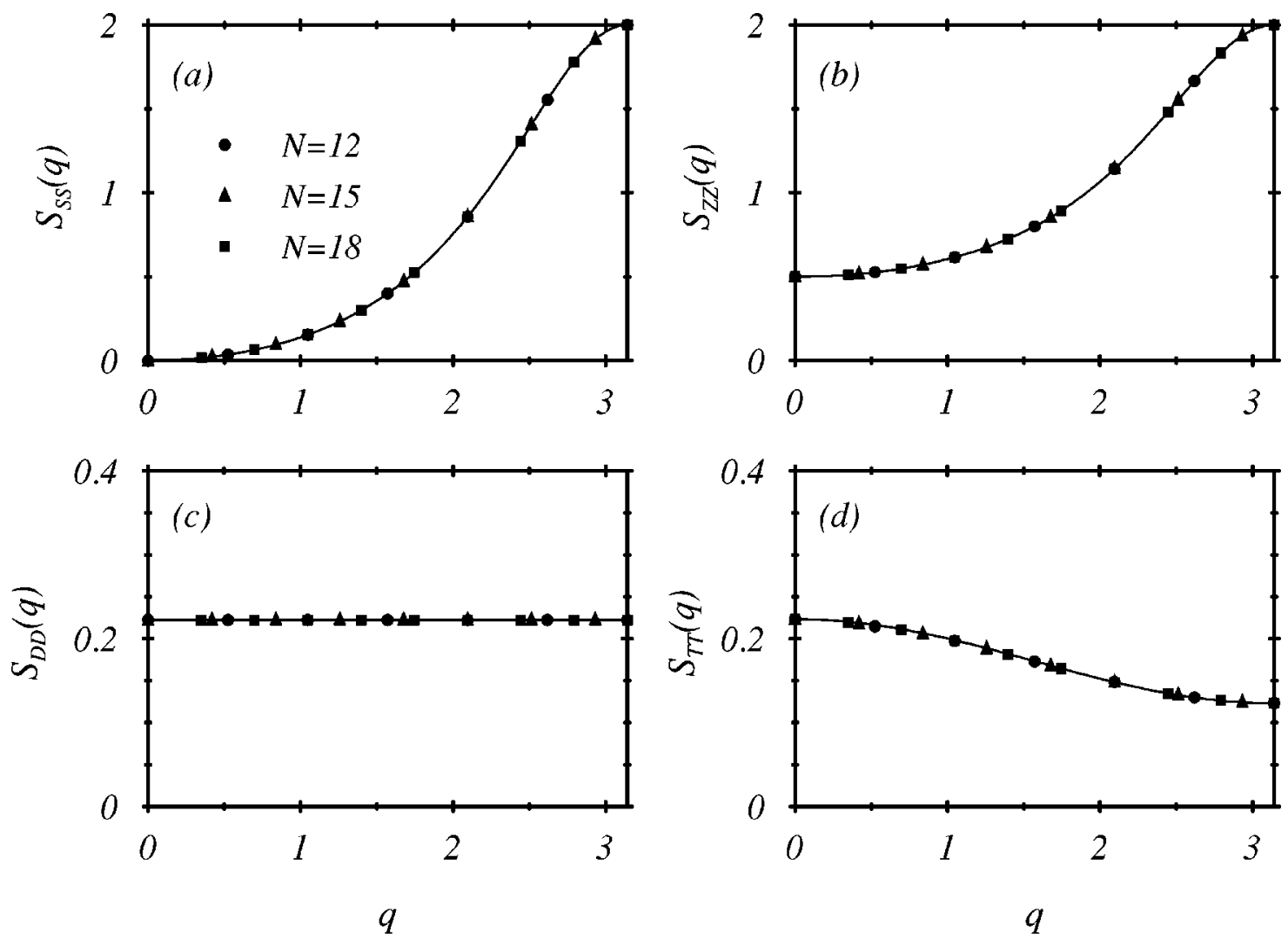

FIG. 1. Static structure factors for the fluctuation operators (a) $F_{q}^{S}$ (spin), (b) $F_{q}^{Z}$ (center), (c) $F_{q}^{D}$ (dimer), (d) $F_{q}^{T}$ (trimer) in the VBS ground state of $H_{\theta}$ at $\theta_{\mathrm{VBS}}=\arctan \frac{1}{3}$ for $N=12(\boldsymbol{O}), N=15(\mathbf{\Delta}), N=18$ (ם) with periodic boundary conditions, and for $N=\infty$ (solid lines).

which is the Laplace transform of the correlation function $\left\langle F_{q}^{A}(t) F_{q}^{A^{\dagger}}\right\rangle$, and from which the dynamic structure factor (1.2) can be directly recovered via the relation

$$
S_{A A}(q, \omega)=2\left\langle F_{q}^{A} F_{q}^{A^{\dagger}}\right\rangle \lim _{\epsilon \rightarrow 0} \operatorname{Re}\left[d_{0}^{A A}(q, \omega+i \epsilon)\right] .
$$

The finite-size continued-fraction analysis expresses the dynamic structure factor in the form

$$
S_{A A}(q, \omega)=\sum_{\lambda} W_{\lambda}^{A} \delta\left(\omega-\omega_{\lambda}^{A}\right),
$$

where the sum runs over all the dynamically relevant excitations $|\lambda\rangle$ with frequency $\omega_{\lambda}^{A}$ and spectral weight $W_{\lambda}^{A}$ $=2 \pi\left|\left\langle G\left|F_{q}^{A}\right| \lambda\right\rangle\right|^{2}$. For the excitations which carry the bulk of the spectral weight both $\omega_{\lambda}^{A}$ and $W_{\lambda}^{A}$ can be extracted quite accurately from a finite number of continued-fraction coefficients.

We begin our study of the static and dynamic structure factors of $H_{\theta}$ at the VBS point, where each of the four ordering tendencies introduced previously (Néel, dimer, trimer, center) is imperceptibly weak, and where some relevant static quantities are exactly known.

\section{VBS STATE}

\section{A. Static structure factors}

The ground-state wave function of $H_{\theta}$ at $\theta=\arctan \frac{1}{3}$ can be constructed for arbitrary $N$ as follows: ${ }^{8-10}$ The spin 1 at each lattice site is expressed as a spin-1/2 pair in a triplet state. The singlet-pair forming valence bond involves one fictitious spin 1/2 from each of two neighboring lattice sites. The VBS state can then be regarded as a chain of valence bonds linking successive spin-1/2 pairs in this manner.

The spin, ${ }^{8,10}$ dimer, ${ }^{18}$ trimer, and center order-parameter correlation functions and the associated static structure factors can be determined exactly:

$$
\begin{gathered}
\left\langle S_{l}^{z} S_{l+n}^{z}\right\rangle=\frac{2}{3}\left(\frac{2(-1)^{n}}{3^{|n|}}-\delta_{n 0}\right), \\
S_{S S}(q)=\frac{2(1-\cos q)}{5+3 \cos q} ; \\
\left\langle D_{l} D_{l+n}\right\rangle=\frac{2}{9} \delta_{n 0}, \quad S_{D D}(q)=\frac{2}{9} ; \\
\left\langle T_{l} T_{l+n}\right\rangle=\frac{\sqrt{12}}{20} \delta_{0 l}+\frac{1}{40}\left(\delta_{1 l}+\delta_{-1 l}\right), \\
S_{T T}(q)=\frac{1}{20}(\sqrt{12}+\cos q) ;
\end{gathered}
$$

$$
\left\langle Z_{l}^{\dagger} Z_{l+n}\right\rangle=\frac{(-1)^{n}}{3^{|n|}}, \quad S_{Z Z}(q)=\frac{4}{5+3 \cos q}
$$

Figure 1 shows finite- $N$ data and the exact result for $N$ 

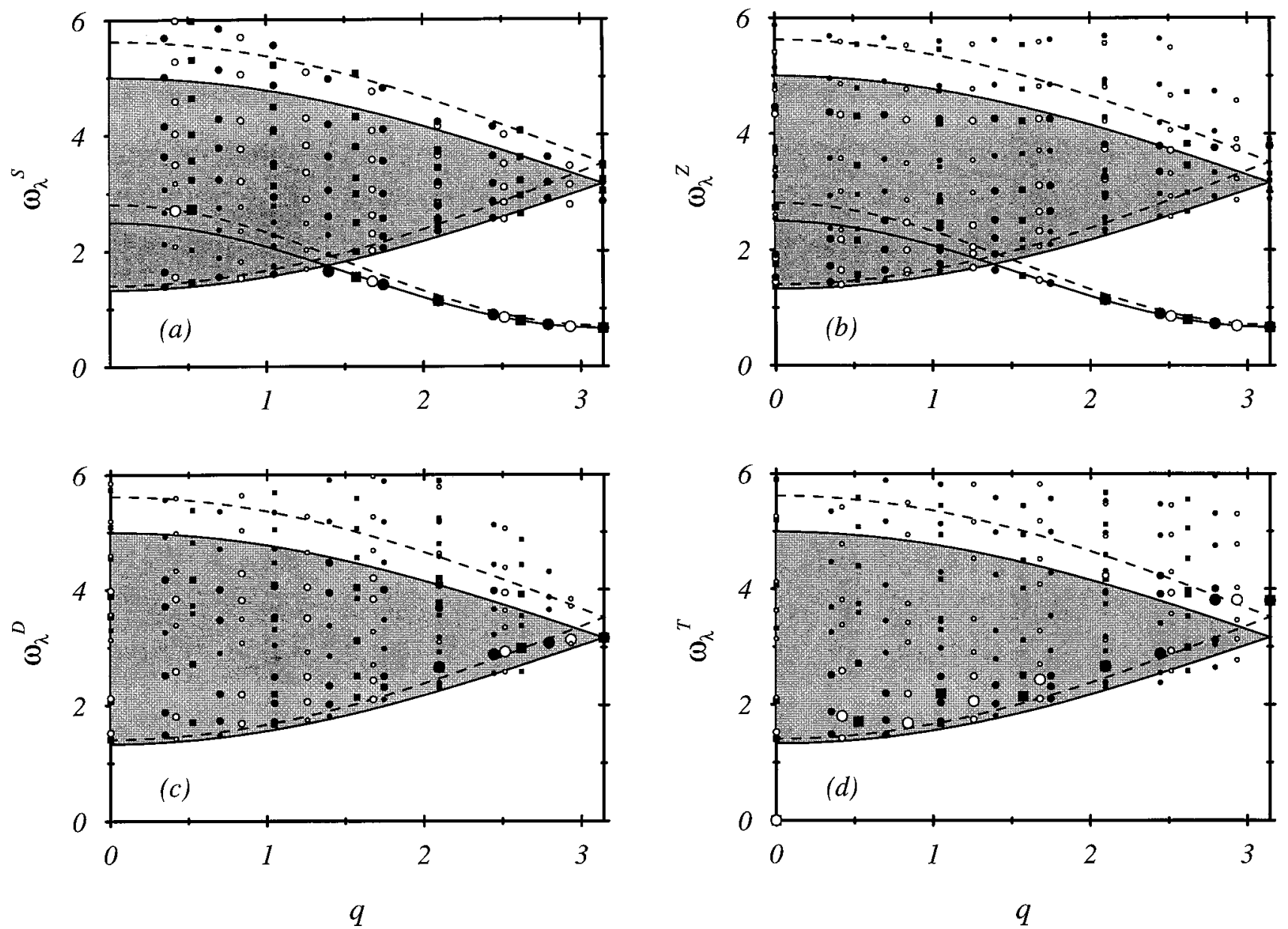

FIG. 2. Frequency $\omega_{\lambda}^{A}$ versus wave number $q$ of the finite- $N$ excitations $[N=12(\boldsymbol{O}), N=15(\bigcirc), N=18(\boldsymbol{\square})]$ which carry most of the spectral weight in the $T=0$ dynamic structure factors $S_{A A}(q, \omega)$ for (a) the spin, (b) the center, (c) the dimer, and (d) the trimer fluctuations of $H_{\theta}$ with $J=1$ at $\theta=\arctan \frac{1}{3}$. The three sizes of symbols used distinguish excitations with relative spectral weight in the ranges $w_{\lambda}^{A} \geqslant 0.5$ (large), $0.5>w_{\lambda}^{A} \geqslant 0.1$ (medium), $0.1>w_{\lambda}^{A} \geqslant 0.001$ (small). The solid and dashed lines represent one-magnon dispersions and two-magnon continuum boundaries as explained in the text.

$=\infty$ of each static structure factor. At the $q$ values realized for $N=12,15,18$, only the trimer data [panel (d)] are subject to finite-size corrections.

At the VBS point, no trace exists of any of the four ordering tendencies which become important in one or the other part of the parameter space. The dimer and trimer correlations are zero for distances $|n| \geqslant 1$ and $|n| \geqslant 2$, respectively, while the spin and center correlations are nonzero but have a very short correlation length $(\xi=\ln 3=1.0986 \ldots$. . . The topological long-range order in the VBS state, ${ }^{19,4}$ described by a string correlation function, is a different matter not discussed here.

\section{B. Dynamic structure factors}

The topological long-range order known to be strongest in the VBS state provides an environment, as we shall see, where pointlike elementary excitations can propagate freely and corresponding stationary states (magnons) form a branch with well-defined dispersion. In the following, these elementary excitations and several kinds of composite excitations made of magnon pairs will be probed from different angles by the four fluctuation operators $F_{q}^{A}$.

In Fig. 2 we display $\omega_{\lambda}^{A}$ versus $q$ of the dynamically relevant spin, center, dimer, and trimer excitation spectra as obtained from the finite-size continued-fraction analysis for $N=12,15,18 .{ }^{11,12}$ The relative spectral weight $w_{\lambda}^{A}$ $\equiv W_{\lambda}^{A} / S_{A A}(q)$ is indicated by the size of the data points. All four spectra are different from each other, but the spin and center spectra [panels (a) and (b)] share some features as do the dimer and trimer spectra [panels (c) and (d)]. The commonalities and differences yield important clues about the composition of each spectrum.

The low-frequency region at $q \gtrsim \pi / 2$ in the spin and center spectra is dominated by a branch of one-magnon states, which have $S_{T}=1$ and, therefore, do not make their appearance in the dimer and trimer spectra. At $q \leqq \pi / 2$ the onemagnon states overlap in energy with what will be identified as a continuum of two-magnon states. Here the magnon interaction precludes the observation of individual onemagnon states. Outside the region of overlap, i.e., for $q$ 
$\gtrless \pi / 2$, the one-magnon states carry more than $95 \%$ of the spectral weight in $S_{S S}(q, \omega)$ and $S_{Z Z}(q, \omega)$.

\section{Single-mode approximation}

An exact single-mode spectrum at any given $q$ value would lead to a spontaneous termination of the recursion algorithm in the first iteration. Terminating it forcibly by setting $b_{1}^{2}(q)=0$ in Eq. (2.8) is equivalent to invoking the single-mode approximation. In general, this is a dubious scheme, but it may have some merit if the spectral-weight distribution is known to be dominated by a single mode. In the present context, the exact calculation of the first continued-fraction coefficient in Eq. (2.8) yields the following single-mode dispersion of the VBS magnons: ${ }^{10}$

$$
\omega_{M}^{(\mathrm{SM})}(q)=J \frac{\sqrt{10}}{9}(5+3 \cos q) .
$$

This prediction, which is shown as a dashed curve in panels (a) and (b) of Fig. 2, fits the finite- $N$ data less than perfectly. The value $\omega_{M}^{(\mathrm{SM})}(\pi) / J=0.70272 \ldots$, for example, exceeds the value $\omega_{M}(\pi) / J=0.66433(2)$ of the extrapolated ${ }^{20}$ finite-size spin excitation energy by more than $5 \%$.

There are three kinds of two-magnon scattering states formed by pairs of one-magnon triplets: states with $S_{T}=1$, which contribute to $S_{S S}(q, \omega)$ and $S_{Z Z}(q, \omega)$, states with $S_{T}$ $=0$, which contribute to $S_{D D}(q, \omega)$ and $S_{T T}(q, \omega)$, and states with $S_{T}=2$, which are observable in $S_{Z Z}(q, \omega)$ only. The magnon interaction is different in each $S_{T}$ subspace. Free two-magnon states form a two-parameter continuum

$$
\omega_{2 M}(k, q) \equiv \omega_{M}(q / 2-k)+\omega_{M}(q / 2+k),
$$

in $(q, \omega)$ space. The continuum boundaries are determined by the solution of $\partial \omega_{2 M} / \partial k=0$. The boundaries thus predicted by the single-mode dispersion (3.5),

$$
\left.\omega_{ \pm}^{(\mathrm{SM})}(q) \equiv \omega_{2 M}^{(\mathrm{SM})}(k, q)\right|_{k=0, \pi}=\frac{2 \sqrt{10}}{9} J\left(5 \pm 3 \cos \frac{q}{2}\right)
$$

are shown dashed in all four panels of Fig. 2. The coalescence of this two-magnon continuum into one spectral line at $q=\pi$ is a consequence of the nongeneric symmetry property

$$
\omega_{M}(q)+\omega_{M}(\pi-q)=\mathrm{const}
$$

of the single-mode one-magnon dispersion $\omega_{M}^{(\mathrm{SM})}(q)$.

\section{Improved one-magnon dispersion}

The key to a significant improvement of the one-magnon dispersion and the two-magnon continuum boundaries over the single-mode predictions (3.5) and (3.7) can be found in the dimer spectrum, i.e., in Fig. 2(c). This spectrum does indeed collapse into a single spectral line at $q=\pi$. The finite- $N$ analysis demonstrates beyond ambiguity that there exists an exact eigenstate, $|D\rangle=F_{\pi}^{D}|G\rangle$ with an $N$-independent excitation energy $\omega_{D}=\sqrt{10} J$, which carries all the spectral weight of $S_{D D}(\pi, \omega)$. We interpret this to be a noninteracting two-magnon singlet. ${ }^{21}$ Its energy is significantly lower than the single-mode prediction, $\omega_{ \pm}^{(\mathrm{SM})}(\pi)$ $=(10 / 9) \sqrt{10} J$.

We now use the exact two-magnon excitation energy, $\omega_{ \pm}(\pi)=\omega_{D}$, in conjunction with the extrapolated value, $\omega_{M}(\pi)=0.66433(2) J$, of the one-magnon excitation gap to construct a modified one-magnon dispersion of the form

$$
\omega_{M}(q)=J(a+b \cos q)
$$

with $a=\sqrt{5 / 2}=1.58113 \ldots$ and $b=0.91681(2)$, which still satisfies the symmetry property (3.8). This dispersion is shown as a solid line in panels (a), (b) of Fig. 2 and the resulting two-magnon continuum (3.6) with boundaries

$$
\omega_{ \pm}(q)=2 J\left(a \pm b \cos \frac{q}{2}\right)
$$

as a shaded region in panels (a) $-(\mathrm{d})$. The finite- $N$ data for the one-magnon states are fitted almost perfectly by Eq. (3.9). The energies of all $N=18$ states that can be clearly identified as one-magnon excitations are listed in Table I. The relative deviation of the single-mode prediction (3.5) and the improved one-magnon dispersion (3.9) from these finite- $N$ data are listed for comparison.

The lower two-magnon boundary $\omega_{-}(q)$ is now in much better agreement with the finite- $N$ two-magnon spectral thresholds in the invariant subspaces with $S_{T}=0,1,2$. Only for $q \lesssim \pi$ do we observe finite- $N$ excitations with significant spectral weight which stray below the predicted two-magnon region in all four panels. Here the spectral threshold of the three-magnon continuum as inferred from Eq. (3.9) is lower than the two-magnon continuum, with a minimum energy $3 \omega_{M}(\pi) / J \simeq 2.0$ at $q=\pi$. However, since the stray states are located near the lower two-magnon boundary, it is more likely that they are interacting two-magnon states than threemagnon states. The nonzero two-magnon bandwidth at $q$ $=\pi$ in the $S_{T}=1$ subspace as observed in the finite- $N$ data of panels (a) and (b) is perhaps the most compelling evidence of the magnon interaction in this region of $(q, \omega)$ space.

A curious phenomenon occurs in the $S_{T}=0$ spectrum of the trimer fluctuations as shown in panel (d). At $q \neq \pi$ all dynamically relevant excitations are identical to those observed in the dimer fluctuations, understandably with different matrix elements. At $q=\pi$, the trimer spectrum collapses into a single spectral line as does the dimer spectrum, but the

TABLE I. One-magnon excitation energies of $H_{\theta}$ at $\theta$ $=\arctan \frac{1}{3}$ for wave numbers $q=(2 \pi / N) \lambda$ and relative deviation of the predictions (3.5) and (3.9).

\begin{tabular}{cccc}
\hline \hline$\lambda$ & \multicolumn{1}{c}{$\omega_{\lambda} / J$} & $\omega_{M}^{(S M)}(q) / \omega_{\lambda}-1$ & $\omega_{M}(q) / \omega_{\lambda}-1$ \\
\hline 3 & $2.092(2)$ & 0.091 & -0.025 \\
4 & $1.649(2)$ & 0.176 & +0.055 \\
5 & $1.4235(2)$ & 0.106 & -0.001 \\
6 & $1.14009(2)$ & 0.079 & -0.015 \\
7 & $0.89071(2)$ & 0.066 & -0.013 \\
8 & $0.72314(2)$ & 0.060 & -0.005 \\
9 & $0.66433(2)$ & 0.058 & Used to fit (3.9) \\
\hline \hline
\end{tabular}


trimer line has a higher energy, $\omega_{T} / J=12 / \sqrt{10}$, which is again $N$ independent. Here we have another exact eigenstate $|T\rangle=F_{\pi}^{T}|G\rangle$. Since $|T\rangle$ is necessarily orthogonal to $|D\rangle$, the former state cannot contribute to the dimer fluctuations, $\left\langle G\left|F_{\pi}^{D}\right| T\right\rangle=0$, and the latter not to the trimer fluctuations, $\left\langle G\left|F_{\pi}^{T}\right| D\right\rangle=0$.

The energy of the state $|T\rangle$ is evidently beyond the range of the two-magnon continuum. If it is formed by two magnons, then it must be a bound state with positive interaction energy. ${ }^{22}$ The data shown in panel (d) indicate that the dominant $S_{T}=2$ contribution to $S_{Z Z}(\pi, \omega)$ comes from a state that is degenerate with the lone trimer excitation $|T\rangle$.

\section{E. Two-magnon scattering states and bound states}

In their density matrix renormalization group (DMRG) study of selected excited states of $H_{\theta}$ for $\theta=0$, White and Huse $^{19}$ observed that at $q$ near $\pi$ the magnon interaction is attractive for two-magnon states with $S_{T}=1$ and repulsive for two-magnon states with $S_{T}=0$ and $S_{T}=2$. The finite- $N$ data for $\theta=\arctan \frac{1}{3}$ in Fig. 2 exhibit trends that are similar in some respects yet different in others.

Near the bottom of the two-magnon region, the interaction is found to be very weak in all three $S_{T}$ subspaces, and neither uniformly attractive nor uniformly repulsive in any $S_{T}$ subspace. At higher energies, the interaction is stronger and repulsive in all three subspaces. The observation that the dynamically relevant excitations in panels (b), (c), (d) spread to higher energies than those in panel (a) indicates that the (positive) interaction energy is considerably larger for $S_{T}$ $=0,2$ than for $S_{T}=1$.

Several excitations, mainly near the bottom of the shaded areas in Fig. 2, can be identified as almost free two-magnon states. There are 28 two-magnon combinations of the onemagnon states listed in Table I. All of them can be found with appreciable spectral weight in at least one of the four excitation spectra within a 5\% margin of interaction energy. In Table II we have listed the excitation energies and interaction energies of these states. Inevitably, some of the assignments made are ambiguous. The uncertainty in any of the excitation energies listed is estimated to be under $0.5 \%$.

The number of states belonging to a two-parameter continuum is of $\mathrm{O}\left(N^{2}\right)$, i.e., of $\mathrm{O}(N)$ for fixed $q$. If the integrated intensity $S_{A A}(q)$ is finite and nonzero for any particular $q$, and if the continuum is to carry a nonzero fraction of the spectral weight in $S_{A A}(q, \omega)$, then the average transition rate of the continuum states must scale like $N^{-1}$. Whereas this particular scaling behavior cannot be verified reliably on the basis of the available data, we have been able to track several of the nearly free two-magnon states at $q=2 \pi / 3$ over the four system sizes $N=9,12,15,18$ and to show that the transition rate tends to converge to zero.

The excitation energy and the relative spectral weight of one such state as observed in the dynamic dimer and trimer structure factors are listed in Table III. ${ }^{23}$

The strong repulsive magnon interaction for $S_{T}=0$ and $S_{T}=2$ as indicated by our data, raises the possibility that discrete branches of bound two-magnon states split off the top of the two-magnon continuum in these two subspaces.

Comparing panels (a) and (b) of Fig. 2 at frequencies 3 $\lessgtr \omega / J \lessgtr 5$, we see that the dynamically relevant finite- $N$ ex-
TABLE II. Selected excitations with energy $\omega_{\lambda_{1} \lambda_{2}}^{A}$ and wave number $q$ in the spin, center, dimer, and trimer spectra of $H_{\theta}$ with $J=1, \quad \theta=\arctan \frac{1}{3}$ for $N=18$ in comparison with the corresponding free two-magnon combinations at energy $\omega_{\lambda_{1}}+\omega_{\lambda_{2}}$ and the same wave number.

\begin{tabular}{|c|c|c|c|c|c|}
\hline$\lambda_{1} \lambda_{2}$ & $\omega_{\lambda_{1}}+\omega_{\lambda_{2}}$ & $q N / 2 \pi$ & $\omega_{\lambda_{1} \lambda_{2}}^{S}$ & $\omega_{\lambda_{1} \lambda_{2}}^{Z}$ & $\omega_{\lambda_{1} \lambda_{2}}^{D, T}$ \\
\hline 99 & 1.329 & 0 & & 1.35 & \\
\hline 89 & 1.387 & 1 & 1.39 & & \\
\hline 88 & 1.446 & 2 & & 1.47 & 1.48 \\
\hline 79 & 1.555 & 2 & 1.55 & & \\
\hline 69 & 1.804 & 3 & 1.75 & 1.76 & \\
\hline 78 & 1.614 & 3 & 1.60 & 1.64 & \\
\hline 59 & 2.088 & 4 & & & 2.01 \\
\hline 68 & 1.863 & 4 & 1.94 & 1.92 & \\
\hline 77 & 1.781 & 4 & & & 1.81 \\
\hline 49 & 2.314 & 5 & & 2.31 & 2.31 \\
\hline 58 & 2.147 & 5 & & 2.09 & 2.10 \\
\hline 67 & 2.031 & 5 & 2.06 & & \\
\hline 39 & 2.757 & 6 & 2.78 & & \\
\hline 48 & 2.373 & 6 & & & 2.37 \\
\hline 57 & 2.314 & 6 & 2.35 & 2.35 & \\
\hline 66 & 2.280 & 6 & & & 2.30 \\
\hline 33 & 4.185 & 6 & 4.15 & & \\
\hline 38 & 2.816 & 7 & 2.85 & & \\
\hline 47 & 2.540 & 7 & & & 2.55 \\
\hline 56 & 3.073 & 7 & & 2.97 & \\
\hline 34 & 3.742 & 7 & & 3.78 & \\
\hline 37 & 2.983 & 8 & & & 3.02 \\
\hline 46 & 2.790 & 8 & & & 2.65 \\
\hline 55 & 2.847 & 8 & 2.91 & 2.92 & \\
\hline 35 & 3.516 & 8 & 3.62 & & \\
\hline 44 & 3.299 & 8 & & 3.33 & \\
\hline 36 & 3.233 & 9 & 3.19 & 3.20 & \\
\hline 45 & 2.564 & 9 & 2.44 & 2.44 & \\
\hline
\end{tabular}

citations are arranged in contrasting patterns. In panel (a) we have an arrangement of points which is typical of a twoparameter continuum. As $N$ increases, more points are added and spread roughly evenly along the frequency axis. In panel (b), by contrast, the data points are arranged in branches with an almost $N$-independent separation.

The number of states belonging to a discrete branch is of $\mathrm{O}(N)$, i.e., one state per wave number over the range of the branch. The transition rate of such a state will converge to a nonzero value as $N \rightarrow \infty$ for any branch that carries a nonzero fraction of the integrated intensity. This convergence can be

TABLE III. Excitation energy and relative spectral weight of one finite- $N$ two-magnon continuum state as observed in $S_{D D}(2 \pi / 3, \omega)$ and $S_{T T}(2 \pi / 3, \omega)$.

\begin{tabular}{rcccc}
\hline \hline$N$ & $\omega_{\lambda}^{D}$ & $w_{\lambda}^{D}$ & $\omega_{\lambda}^{T}$ & $w_{\lambda}^{T}$ \\
\hline 9 & 2.34 & 0.112 & 2.34 & 0.249 \\
12 & 2.31 & 0.042 & 2.31 & 0.095 \\
15 & 2.30 & 0.020 & 2.30 & 0.046 \\
18 & 2.30 & 0.015 & 2.30 & 0.031 \\
\hline \hline
\end{tabular}


TABLE IV. Excitation energy and spectral weight as observed in $S_{Z Z}(2 \pi / 3, \omega), S_{D D}(2 \pi / 3, \omega)$, or $S_{T T}(2 \pi / 3, \omega)$ of several finite$N$ excited states that seem to belong to discrete branches in the limit $N \rightarrow \infty$.

\begin{tabular}{rcccccc}
\hline \hline \multicolumn{1}{c}{$N$} & $\omega_{\lambda}^{Z}$ & $w_{\lambda}^{Z}$ & $\omega_{\lambda}^{D}$ & $w_{\lambda}^{D}$ & $\omega_{\lambda}^{T}$ & $w_{\lambda}^{T}$ \\
\hline 9 & 3.259 & 0.050 & 2.723 & 0.486 & 2.723 & 0.475 \\
12 & 3.369 & 0.082 & 2.654 & 0.540 & 2.654 & 0.611 \\
15 & 3.215 & 0.112 & 2.654 & 0.525 & 2.654 & 0.595 \\
18 & 3.265 & 0.117 & 2.661 & 0.532 & 2.660 & 0.594 \\
9 & 3.571 & 0.151 & 4.317 & 0.150 & 4.317 & 0.181 \\
12 & 3.786 & 0.108 & 4.196 & 0.160 & 4.179 & 0.132 \\
15 & 3.736 & 0.110 & 4.114 & 0.185 & 4.233 & 0.108 \\
18 & 3.812 & 0.114 & 4.078 & 0.193 & 4.313 & 0.091 \\
\hline \hline
\end{tabular}

demonstrated most convincingly for the one-magnon state at $q=2 \pi / 3$, because it is far removed in energy from any other state in the same invariant subspace. Our data for $N$ $=9,12,15,18$ yield $\omega_{\lambda} / J \rightarrow 1.14009(1), w_{\lambda}^{S} \rightarrow 0.95124(1)$, $w_{\lambda}^{Z} \rightarrow 0.53507(2)$.

Several higher lying states in $S_{Z Z}(2 \pi / 3, \omega)$ which appear to be part of discrete branches have been tracked over the system sizes $N=9,12,15,18$. Their transition rates also tend to converge to a nonzero value. The data for two such states are compiled in Table IV.

The arrangement of points in panels (c) and (d) of Fig. 2 looks more irregular. Evidence for the layered structure typical of discrete branches can be discerned at high frequencies $(\omega / J \gtrsim 5)$. At lower frequencies, some of the dynamically relevant states have already been identified as nearly free two-magnon continuum states. But then we can also observe states with a fairly large spectral weight whose $N$ dependence indicates that they belong to a discrete branch.

The most prominent such branch in panel (c) has its endpoint at $q=\pi$ in the exact dimer excitation $|D\rangle$ discussed previously. A corresponding branch which ends in the exact trimer excitation $|T\rangle$ at $q=\pi$ can be discerned in panel (d).

It may well be the case that the wave function of the eigenstate $|T\rangle=F_{\pi}^{T}|G\rangle$ has bound-state character with positive interaction energy and the eigenstate $|D\rangle=F_{\pi}^{D}|G\rangle$ scattering-state character (with zero interaction energy). A clearcut distinction between the wave functions of bound states and scattering states is known to exist even for finite $N$ in the $s=1 / 2$ Heisenberg ferromagnet, for example, and can be described in the framework of the Bethe ansatz. ${ }^{24}$

\section{HALDANE PHASE}

\section{A. Static structure factors}

Any change of the Hamiltonian parameter $\theta$ away from the value $\theta_{\mathrm{VBS}}$ in the interior of the Haldane phase toward lower or higher values softens the topological long-range order and thus enhances specific quantum fluctuations in the ground state. The contrasting enhancements of fluctuations for decreasing or increasing $\theta$ values, which reflect the different ordering tendencies near the predicted phase boundaries at $\theta=-\pi / 4$ and $\theta \lesssim \pi / 4$, respectively, can be observed to a certain extent in the finite- $N$ static structure factors for the spin, center, dimer, and trimer correlations, as shown in
Fig. 3. The finite- $N$ effects are minute except for $q=2 \pi / 3$ at $\theta \simeq \pi / 4$ and for $q=\pi$ at $\theta<0$.

In the long-wavelength limit $q \rightarrow 0$, the function $S_{S S}(q)$ is observed to vanish for all $\theta$ values covered in Fig. 3, whereas $S_{D D}(q)$ vanishes only at $\theta=0, S_{Z Z}(q)$ only at $\theta=-\pi / 4$, and $S_{T T}(q)$ not at all. These properties are the result of different conservation laws:

The $z$ component of the total spin, $S_{T}^{z} \equiv \Sigma_{l} S_{l}^{z}$, is a constant of the motion for all $\theta$ values, and it has eigenvalue zero in the singlet ground state. At $\theta=0$, the operator $D_{T}$ $\equiv \Sigma_{l} D_{l}$ with $D_{l}$ as defined in Eq. (2.3) can be written as $D_{T}=\left(H-E_{0} / J\right)$ and is, therefore, a constant of the motion with zero eigenvalue.

The operator $Z_{T} \equiv \Sigma_{l} Z_{l}$ with $Z_{l}$ as defined in Eq. (2.7) commutes with $H_{\pi / 4}$. Furthermore, the Bethe ansatz demonstrates that all eigenvectors of $H_{\pi / 4}$ have definite numbers $n_{1}, n_{2}, n_{3}$ of up, zero, and down spins, respectively, on the $N$ sites of the lattice. The ground state has $n_{1}=n_{2}=n_{3}$ $=N / 3$, which implies that $Z_{T}$ has eigenvalue zero.

As $\theta$ decreases from the value $\theta_{\mathrm{VBS}}$, the spin and dimer fluctuations at $q=\pi$ experience a gradual enhancement, which reflects a continuous increase in the spin and dimer correlation lengths. ${ }^{25}$ On approach of the phase boundary at $\theta=-\pi / 4$, these correlation lengths diverge. At $\theta=-\pi / 4$ the spectra with $S_{T}=0,1$ are gapless, and the static spin and dimer structure factors exhibit cusp singularities at $q=\pi$.

At this critical point, the spin and dimer ordering tendencies are in competition. A perturbation of $\mathrm{H}_{-\pi / 4}$ can produce Néel long-range order or dimer long-range order. Uniaxial exchange anisotropy, for example, is likely to produce Néel long-range order, whereas the further strengthening of the biquadratic exchange $(\theta \lesssim-\pi / 4)$ is almost certain to produce dimer long-range order. ${ }^{2}$

In the regime $-\pi / 4 \leqslant \theta \leqslant \theta_{\mathrm{VBS}}$ of the Haldane phase, the center structure factor exhibits properties very similar to those of the spin and dimer structure factors. It remains to be seen whether the $q=\pi$ enhancement in the center structure factor is due primarily due to the $S_{T}=1$ excitations, which also dominate the spin structure factor or whether the $S_{T}$ $=2$ spectrum contributes significantly to the spectral intensity. The trimer structure factor, by contrast, remains featureless throughout this regime. The conclusion is that there are no significant trimer fluctuations in this part of the phase diagram.

In the regime $\theta_{\mathrm{VBS}} \leqslant \theta \leqslant \pi / 4$, it is the dimer fluctuations that remain insignificant and the dimer structure factor that remains featureless. Here the prevailing ordering tendencies are captured by the spin, center and trimer structure factors. In all three of them a distinct enhancement in intensity at $q$ $=2 \pi / 3$ emerges as $\theta$ increases toward the Lai-Sutherland point $\theta=\pi / 4$.

The DMRG study of Schollwöck et al. ${ }^{4}$ showed that as $\theta$ increases from $\theta_{\mathrm{VBS}}$ the spin correlations start to become incommensurate and that the correlation length increases gradually. The underlying periodicity moves gradually from $q=\pi$ at $\theta_{\mathrm{VBS}}$ to $q=2 \pi / 3$ at or before $\theta_{L S}$. Because of the short-range nature of the spin correlations, the change of periodicity in real space is not fully synchronized with the movement of the peak of the static structure factor in reciprocal space. 

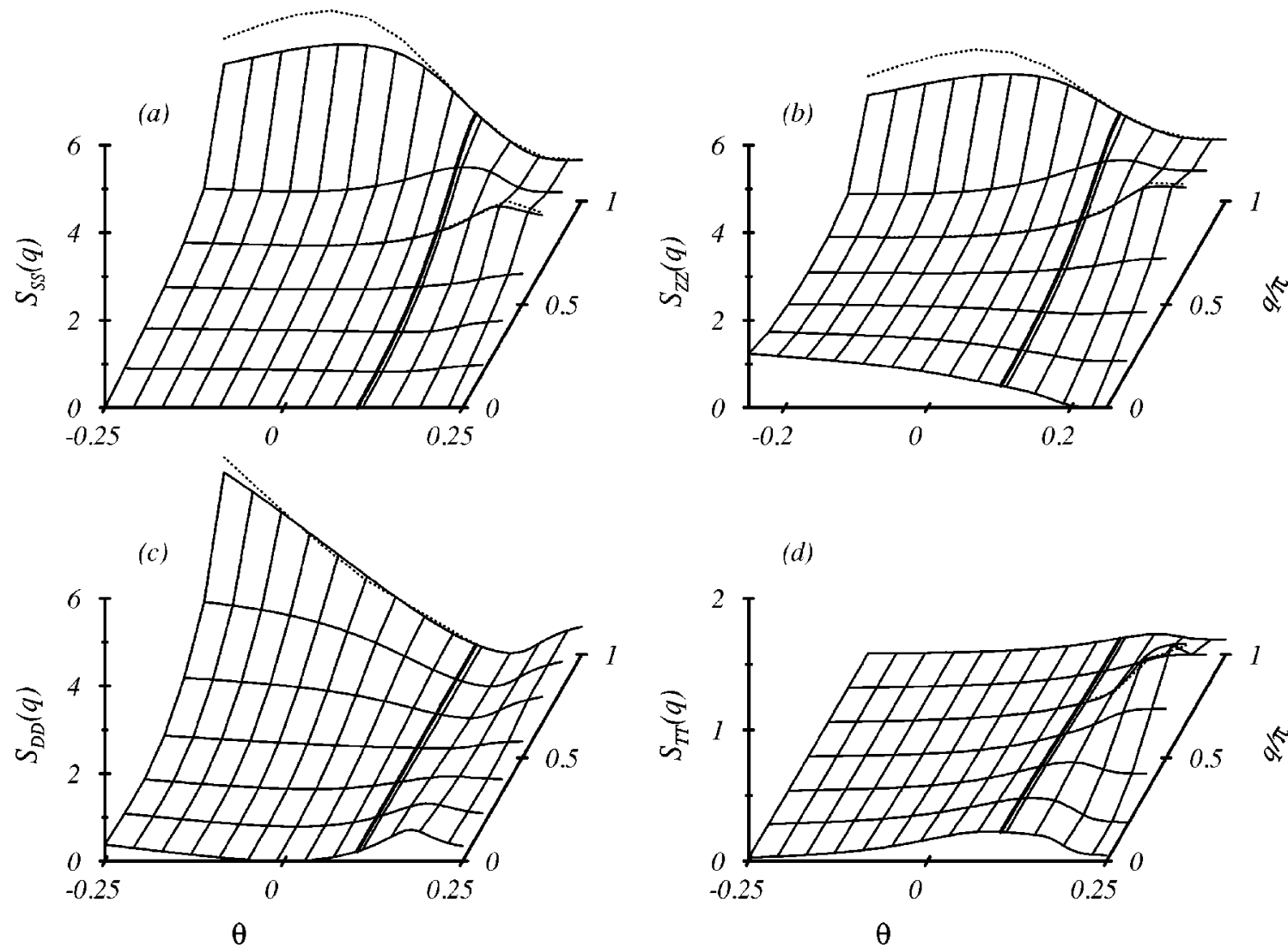

(d)

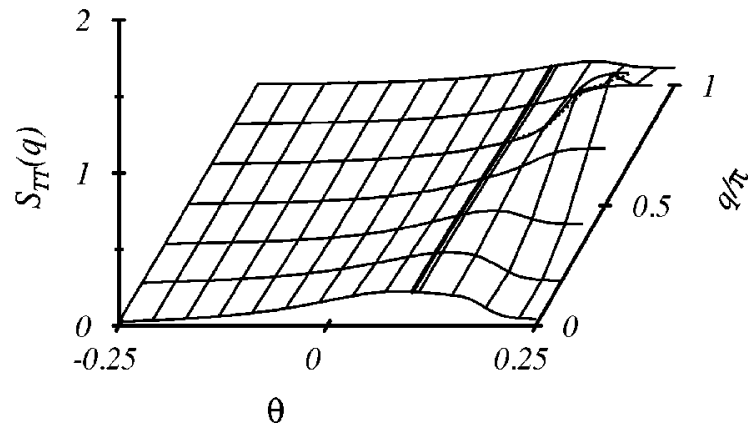

FIG. 3. Static structure factors for the fluctuation operators (a) $F_{q}^{S}$ (spin), (b) $F_{q}^{Z}$ (center), (c) $F_{q}^{D}$ (dimer), (d) $F_{q}^{T}$ (trimer) plotted versus $q$ and $\theta$ for $N=12$ over the range $-\pi / 4 \leqslant \theta \leqslant \pi / 4$. The dotted lines represent $N=18$ data for $q=2 \pi / 3, \pi$.

The phase at $\theta>\pi / 4$ has been named "trimerized" phase with no compelling evidence in support of trimer ordering, mainly because the dominant fluctuations have period three. However, there is evidence that the dominant fluctuations at $q=2 \pi / 3$ are not those that are probed by the trimer fluctuation operator.

One piece of evidence is provided by the $N$ dependence of the static spin, trimer, and center structure factors in the vicinity of the phase boundary at $\theta \lesssim \pi / 4$, whose exact position is not firmly established. These values are listed in Table $\mathrm{V}$. The trimer intensities extrapolate downward, whereas the spin and center intensities extrapolate upward. The highest intensity is observed in the center structure factor. ${ }^{26}$

Further evidence which speaks against the trimer nature of the phase at $\theta>\pi / 4$ can be inferred from the finite- $N$ excitation spectrum at $q=2 \pi / 3$, where the trimer orderparameter fluctuations are expected to become dominant. It

TABLE V. Static spin, trimer, and center structure factors at $q=2 \pi / 3$ for finite- $N$ and extrapolated values (Ref. 20).

\begin{tabular}{rcccccc}
\hline \hline \multirow{2}{*}{$N$} & \multicolumn{2}{c}{$S_{S S}(2 \pi / 3)$} & \multicolumn{2}{c}{$S_{T T}(2 \pi / 3)$} & \multicolumn{2}{c}{$S_{Z Z}(2 \pi / 3)$} \\
$\theta / \pi$ & 0.20 & 0.25 & 0.20 & 0.25 & 0.20 & 0.25 \\
\hline 9 & 1.3386 & 1.2068 & 0.5803 & 0.6288 & 1.8166 & 1.8102 \\
12 & 1.4366 & 1.2507 & 0.5390 & 0.6049 & 1.8899 & 1.8761 \\
15 & 1.5145 & 1.2825 & 0.5151 & 0.5952 & 1.9440 & 1.9237 \\
18 & 1.5778 & 1.3070 & 0.4984 & 0.5906 & 1.9854 & 1.9606 \\
$\infty$ & $2.15(3)$ & $1.76(3)$ & $0.44(2)$ & $0.58(2)$ & $2.28(1)$ & $2.64(5)$ \\
\hline \hline
\end{tabular}

turns out that in this parameter regime the lowest excitation at $q=2 \pi / 3$ is not a state with $S_{T}=0$, which could contribute to $S_{T T}(q, \omega)$, nor is it a state with $S_{T}=1$, which could contribute to $S_{S S}(q, \omega)$. From Ref. 1 we know that it is a state with $S_{T}=2$, and our dynamics data show that this excitation contributes to $S_{Z Z}(q, \omega)$ with a large transition rate.

\section{B. One-magnon and two-magnon states}

The finite- $N$ data for the dynamic spin, center, dimer, and trimer structure factors throughout the Haldane phase indicate that we must distinguish two regimes. For $\theta_{\mathrm{VBS}} \leqslant \theta$ $\leqslant \pi / 4$, the structure of the low-lying excitations as seen through the lenses of all four dynamic structure factors undergoes a major metamorphosis. The analysis of this spectrum and its relation to the prevailing ordering tendency will be the focus of a separate study that uses the exactly solvable case $\theta=\pi / 4$ as the starting point. ${ }^{13}$

Over much of the range $-\pi / 4<\theta<\theta_{\mathrm{VBS}}$, the dynamically relevant spectrum continues to be dominated by onemagnon and two-magnon states with properties that connect seamlessly to the results for $\theta_{\mathrm{VBS}}$ established in Sec. III. However, at $\theta<\theta_{\mathrm{VBS}}$, the two-parameter ansatz (3.9) for the one-magnon dispersion is now inadequate because the symmetry (3.8) is no longer supported by the finite- $N$ data. The absence of this symmetry at $\theta<\theta_{\mathrm{VBS}}$ is also indicated by the manifestly nonzero width at $q=\pi$ of the two-magnon continua.

We propose to use instead the three-parameter ansatz 

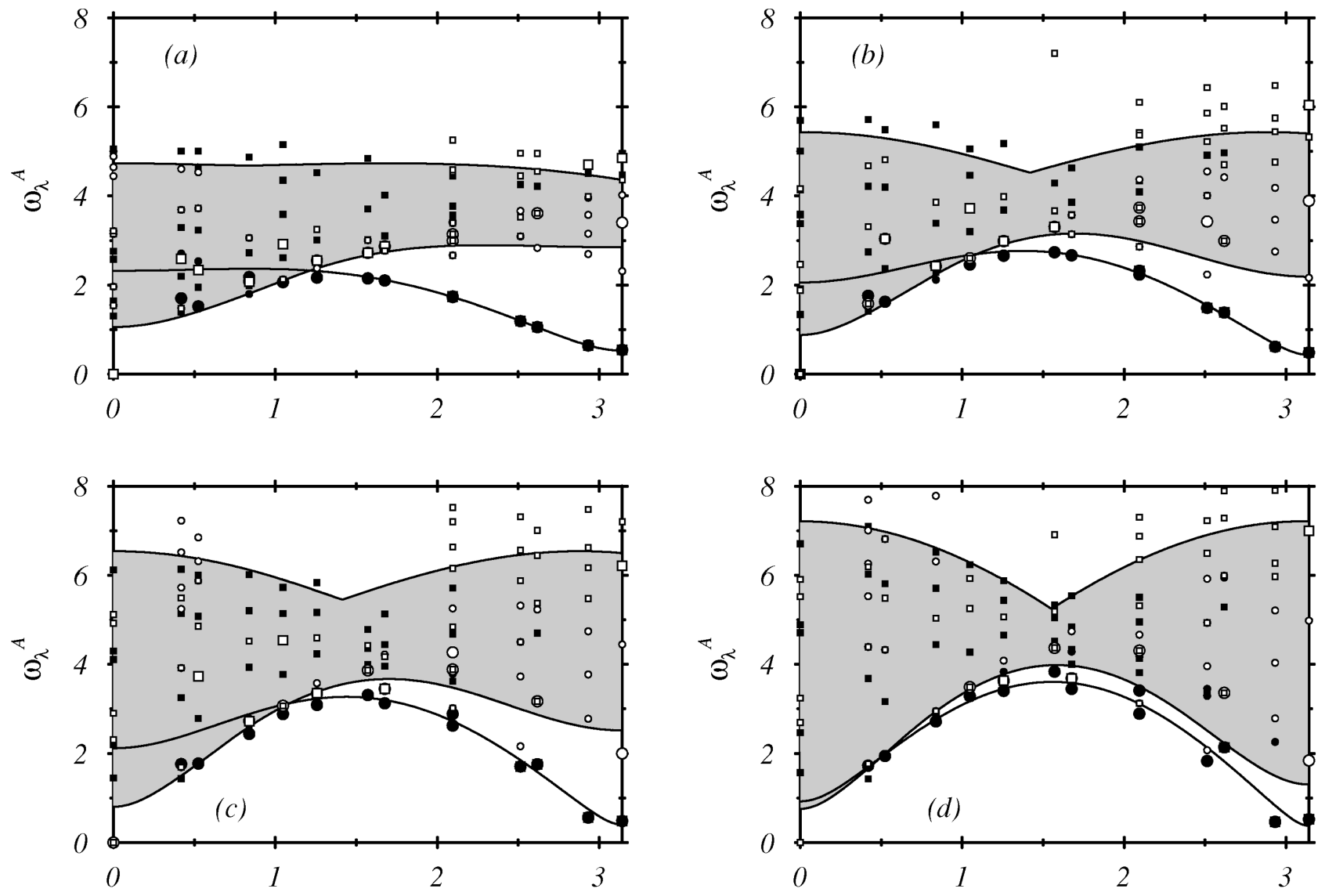

$q$

$q$

FIG. 4. Frequency $\omega_{\lambda}^{A}$ versus wave number $q$ of the finite- $N$ excitations $[N=12$ and $N=15]$ which carry most of the spectral weight in the $T=0$ dynamic structure factors $S_{A A}(q, \omega)$ for the spin $(\bullet)$, center $(\boldsymbol{\square})$, dimer $(\bigcirc)$, and trimer ( $\square$ ) fluctuations of $H_{\theta}$ with $J=1$ at (a) $\theta / \pi=0.05$, (b) $\theta / \pi=0$, (c) $\theta / \pi=-0.05$, (d) $\theta / \pi=-0.10$. The symbol sizes are explained in the caption of Fig. 2 . The solid lines represent the one-magnon dispersion (4.1) and the associated two-magnon boundaries with parameter values (a) $a=2.18, b_{1}=2.09, b_{2}$ $=-0.92$, (b) $a=2.70, b_{1}=2.66, b_{2}=-2.13$, (c) $a=3.25, b_{1}=3.22, b_{2}=-2.55$, (d) $a=3.61, b_{1}=3.58, b_{2}=-3.49$.

$$
\omega_{M}(q)=J \sqrt{\left(a+b_{1} \cos q\right)\left(a+b_{2} \cos q\right)}
$$

with $a>0$ and $\left|b_{1}\right|,\left|b_{2}\right| \leqslant a$ as an interpolation formula between $\theta=\theta_{\mathrm{VBS}}$, where we have $a=\sqrt{5 / 2}, \quad b_{1}=b_{2} \equiv b$ $=0.91681(2)$ as discussed previously, and $\theta=-\pi / 4$, where Eq. (4.1) with $a=b_{1}=-b_{2}=\pi$ describes magnon states having turned into spinon states that are amenable to a rigorous analysis in the framework of the Bethe ansatz. ${ }^{5}$

Interestingly, the three-parameter dispersion is already known to have at least two exact realizations in the dynamics of spin chains. (i) The magnon dispersion of the classical spin-s $X Y Z$ antiferromagnet with $J_{x} \geqslant J_{y} \geqslant J_{z}$ is given by ${ }^{27}$

$$
\omega_{X Y Z}^{\mathrm{CL}}(q)=2 s \sqrt{\left(J_{x}-J_{y} \cos q\right)\left(J_{x}+J_{z} \cos q\right)} .
$$

(ii) The one-particle dispersion in the fermion representation of the $s=1 / 2 X Y$ model with couplings $J_{ \pm}=\frac{1}{2}\left(J_{x} \pm J_{y}\right)$ and magnetic field $h_{z}$ is given by ${ }^{28}$

$$
\omega_{X Y}^{Q U}(q)=\sqrt{\left(h_{z}+J_{+} \cos q\right)^{2}+J_{-}^{2} \sin ^{2} q},
$$

which is, for restricted $h_{z}, J_{ \pm}$, equivalent to Eq. (4.1). In the $X Y$ model, the special case $h_{z}^{2}=J_{+}^{2}-J_{-}^{2}$, where Eq. (4.3) becomes a linear function of $\cos q$, has been identified as a disorder point, where the spin fluctuations are minimally correlated, ${ }^{27,29}$ such as is also the case at the VBS point of the spin-1 chain (1.1).

The deformation of the one-magnon dispersion over the range $-\pi / 4<\theta<\theta_{\mathrm{VBS}}$ has a strong effect on the shape of the associated two-magnon continuum. Of special interest are the excitation gaps at $q=0$ and $q=\pi$. Their dependence on the parameters $a, b_{1}, b_{2}$ is

$$
\begin{gathered}
\omega_{ \pm}(0)=J \sqrt{\left(a \pm b_{1}\right)\left(a \pm b_{2}\right)}, \\
\omega_{+}(\pi)=2 J a \\
\omega_{-}(\pi)=J\left[\sqrt{\left(a+b_{1}\right)\left(a+b_{2}\right)}+\sqrt{\left(a-b_{1}\right)\left(a-b_{2}\right)}\right] .
\end{gathered}
$$

The adequacy of the three-parameter dispersion for the description of the dynamically dominant excitation spectrum must be judged on the two requirements (i) that it yields a reasonable fit of the one-magnon energies and (ii) that the associated two-magnon continuum covers the range of the corresponding finite-chain data, especially near the spectral threshold.

The finite- $N$ data shown in Fig. 4 for four values of $\theta$ 
between $\theta_{\mathrm{VBS}}$ and $-\pi / 4$ confirm that the excitations dominating the $T=0$ dynamical properties in this regime remain well described in terms of a branch one-magnon states and a continuum of two-magnon scattering states.

\section{ACKNOWLEDGMENTS}

The work at URI was supported by NSF Grant DMR-9312252 and by the Max Kade Foundation.

\section{APPENDIX: STATES WITH MAXIMUM NÉEL, DIMER, TRIMER, OR CENTER LONG-RANGE ORDER}

The order parameters associated with the four fluctuation operators $F_{q}^{S}$ (spin), $F_{q}^{D}$ (dimer), $F_{q}^{T}$ (trimer), $F_{q}^{Z}$ (center) introduced in Sec. II can be written in the form

$$
P_{A}=\frac{1}{N} \sum_{l=1}^{N} e^{i q_{A} l} A_{l},
$$

where $q_{S}=q_{D}=\pi$, and $q_{T}=q_{Z}=2 \pi / 3$. Their exact nature is best illustrated by those eigenvectors of each operator $P_{A}$ with an eigenvalue of maximum magnitude. Every such vector $\left|\Phi_{k}^{A}\right\rangle, k=1,2, \ldots$ represents the long-range order associated with order parameter $P_{A}$ in its purest form. For each operator $P_{A}$ there must be at least two such vectors for it to qualify as an order parameter.

(i) There are two Néel states with eigenvalues $\exp \left(i q_{S} k\right)$ :

$$
\left|\Phi_{1}^{S}\right\rangle=|+-+-\cdots\rangle, \quad\left|\Phi_{2}^{S}\right\rangle=|-+-+\cdots\rangle .
$$

(ii) There are two dimer states with eigenvalues $\exp \left(i q_{D} k\right) / 2$ :

$$
\left|\Phi_{1}^{D}\right\rangle=|[12][34] \cdots\rangle, \quad\left|\Phi_{2}^{D}\right\rangle=|[23][45] \cdots\rangle,
$$

where $|[l, l+1]\rangle=(|+-\rangle+|-+\rangle-|00\rangle) / \sqrt{3}$ represents a singlet state formed by two spins 1 on neighboring sites.

(iii) There are three trimer states with eigenvalues $\exp \left(i q_{T} k\right) / 3$ :

$$
\begin{gathered}
\left|\Phi_{1}^{T}\right\rangle=|[123][456] \cdots\rangle, \quad\left|\Phi_{2}^{T}\right\rangle=|[234][567] \cdots\rangle, \\
\left|\Phi_{3}^{T}\right\rangle=|[345][678] \cdots\rangle,
\end{gathered}
$$

where $|[l, l+1, l+2]\rangle$ as given in Eq. (2.6) represents a singlet state formed by three spins 1 on consecutive sites.

(iv) There are six center states with eigenvalues $\exp \left(i q_{Z} k\right)$ :

$$
\begin{gathered}
\left|\Phi_{1}^{Z}\right\rangle=|0-+\cdots\rangle, \quad\left|\Phi_{2}^{Z}\right\rangle=|+0-\cdots\rangle, \\
\left|\Phi_{3}^{Z}\right\rangle=|-+0 \cdots\rangle .
\end{gathered}
$$

All these vectors represent nonstationary symmetrybreaking states in the context of the Hamiltonian (1.1). None of the order parameters $P_{A}$ commutes with $H_{\theta}$. The four types of long-range order are also reflected in the orthonormal linear combinations $\left|A_{k}\right\rangle$ of the vectors $\left|\Phi_{k}^{A}\right\rangle$ which restore the translational symmetry. For $N \rightarrow \infty$ they have the form ${ }^{30}$

$$
\left|S_{k}\right\rangle=\frac{1}{\sqrt{2}}\left(\left|\Phi_{1}^{S}\right\rangle+e^{i q_{S} k}\left|\Phi_{2}^{S}\right\rangle\right),
$$

$$
\begin{gathered}
\left|D_{k}\right\rangle=\frac{1}{\sqrt{2}}\left(\left|\Phi_{1}^{D}\right\rangle+e^{i q_{D} k}\left|\Phi_{2}^{D}\right\rangle\right), \\
\left|T_{k}\right\rangle=\frac{1}{\sqrt{3}}\left(\left|\Phi_{1}^{T}\right\rangle+e^{i q_{T^{k}}}\left|\Phi_{2}^{T}\right\rangle+e^{2 i q_{T^{k}}}\left|\Phi_{3}^{T}\right\rangle\right), \\
\left|Z_{k}\right\rangle=\frac{1}{\sqrt{3}}\left(\left|\Phi_{1}^{Z}\right\rangle+e^{i q_{Z^{k}}}\left|\Phi_{2}^{Z}\right\rangle+e^{2 i q_{Z^{k}}}\left|\Phi_{3}^{Z}\right\rangle\right) .
\end{gathered}
$$

The correlation functions

$$
C_{A A}^{A_{k} A_{k}}(n) \equiv\left\langle A_{k}\left|A_{l} A_{l+n}\right| A_{k}\right\rangle-\left\langle A_{k}\left|A_{l}\right| A_{k}\right\rangle\left\langle A_{k}\left|A_{l+n}\right| A_{k}\right\rangle
$$

in the symmetry-restored eigenvectors of the order parameters are found to be independent of $k$ and exhibit the characteristic asymptotic behavior

$$
C_{A A}^{A_{k} A_{k}}(n) \stackrel{n \rightarrow \infty}{\rightarrow} \cos \left(q_{A} n\right) O_{A}^{2},
$$

if the state $A_{k}$ corresponds to the operator $A$. Here $O_{A}$ is the saturation value of the order parameter $P_{A}: O_{S}^{2}=1, O_{D}^{2}$ $=1, O_{T}=2(2 / 3)^{6}, O_{Z}=1$. In all cases it turns out that the asymptotic behavior is reached already at small distances $(|n| \geqslant 3)$.

If the state $A_{k}$ does not correspond to the operator $A$ in Eq. (A10), such as in $C_{D D}^{S_{k} S_{k}}(n)$, then these correlation functions are found to vanish for all pairs of spins with $|n| \geqslant 3$. There are two exceptions to that rule:

$$
\begin{aligned}
& C_{S S}^{Z_{k} Z_{k}(n)}=\frac{2}{3} \cos \left(q_{Z} n\right) \\
& C_{Z Z}^{S_{k} S_{k}}(n)=\frac{3}{4} \cos \left(q_{S} n\right) .
\end{aligned}
$$

This anomaly tells us that the spin-fluctuation operator $F_{q}^{S}$ does not only probe Néel long-range order (at $q=\pi$ ) but also center long-range order (at $q=2 \pi / 3$ ) as defined in this paper. Likewise, the center fluctuation operator $F_{q}^{Z}$, designed here to probe a particular type of fluctuation and potential ordering, does not only see center long-range order (at $q$ $=2 \pi / 3$ ) when such order exists but also Néel long-range order (at $q=\pi$ ) when that is present.

The main purpose of introducing the center fluctuation operator, which couples to the $S_{T}=1$ and $S_{T}=2$ excitation spectra, has been to illuminate aspects of the predominant fluctuations in the parameter range $\pi / 4<\theta<\pi / 2$, which are invisible to any of the other three fluctuation operators, which all couple either to the $S_{T}=1$ spectrum (spin) or to the $S_{T}=0$ spectrum (dimer, trimer). This distinctive property of $F_{q}^{Z}$ is of vital importance in view of the fact that the lowestlying finite- $N$ excitations in this parameter range have total spin $S_{T}=2$. It may well be the case that a different fluctuation operator, which does not see the Néel long-range order and whose characteristic long-range order is not picked up by the spin-fluctuation operator provides a better description of the predominant fluctuations in this regime. However, a suitable candidate has yet to be found. 
Nontrivial realizations of the fully ordered ground states are known at least three of the four order parameters: (i) The Néel states (A2) are stabilized in the 1D $s=1 / 2 X Y Z$ model with $0<J_{x}=-J_{y}<J_{z} \cdot{ }^{31}$ (ii) The $s=1$ dimer states (A3) have $s=1 / 2$ counterparts, which are constructed from corresponding singlet states: $|[l, l+1]\rangle=(|\uparrow \downarrow\rangle-|\downarrow \uparrow\rangle) / \sqrt{2}$. The dimer ground states are realized in the $s=1 / 2$ Hamiltonian $^{32,9}$

$$
H=\sum_{n=1}^{N}\left(\mathcal{P}_{n, n+1}+\frac{1}{2} \mathcal{P}_{n, n+2}\right),
$$

with nearest and next-nearest neighbor interactions, here expressed in terms of permutation operators

$$
\mathcal{P}_{n, m}=\frac{1}{2}+2 \mathbf{S}_{n} \cdot \mathbf{S}_{m}
$$

*Present address: Department of Physics, University of Wuppertal, D-42097 Wuppertal, Germany.

${ }^{1}$ G. Fáth and J. Sólyom, Phys. Rev. B 44, 11836 (1991).

${ }^{2}$ G. Fáth and J. Sólyom, Phys. Rev. B 51, 3620 (1995).

${ }^{3}$ R. J. Bursill, T. Xiang, and G. A. Gehring, J. Phys. A 28, 2109 (1995).

${ }^{4}$ U. Schollwöck, T. Jolicoeur, and T. Garel, Phys. Rev. B 53, 3304 (1996).

${ }^{5}$ L. A. Takhtajan, Phys. Lett. 87A, 479 (1982); H. M. Babujian, ibid. 90A, 479 (1982); H. M. Babujian, Nucl. Phys. B 215, 317 (1983).

${ }^{6}$ G. V. Uimin, JETP Lett. 12, 225 (1970); C. K. Lai, J. Math. Phys. 15, 1675 (1974); B. Sutherland, Phys. Rev. B 12, 3795 (1975).

${ }^{7}$ J. B. Parkinson, J. Phys. C 20, L1029 (1987); 21, 3793 (1988); M. N. Barber and M. T. Batchelor, Phys. Rev. B 40, 4621 (1988); A. Klümper, Europhys. Lett. 9, 815 (1989); A. Klümper, J. Phys. A 23, 809 (1990); E. Sørsensen and A. P. Young, Phys. Rev. B 42, 754 (1990).

${ }^{8}$ I. Affleck, T. Kennedy, E. H. Lieb, and H. Tasaki, Phys. Rev. Lett. 59, 799 (1987)

${ }^{9}$ I. Affleck, T. Kennedy, E. H. Lieb, and H. Tasaki, Commun. Math. Phys. 115, 477 (1988).

${ }^{10}$ D. P. Arovas, A. Auerbach, and F. D. M. Haldane, Phys. Rev. Lett. 60, 531 (1988)

${ }^{11}$ V. S. Viswanath and G. Müller, The Recursion Method, LNP m23 (Springer-Verlag, Berlin, 1994).

${ }^{12}$ A. Fledderjohann, M. Karbach, K.-H. Mütter, and P. Wielath, J. Phys.: Condens. Matter 7, 8993 (1995).

${ }^{13} \mathrm{M}$. Karbach and G. Müller (unpublished).

${ }^{14}$ The subtraction of constants in operators (2.3) and (2.4) removes artificial contributions $\propto \delta_{q, 0}$ from the dimer and trimer structure factors, and thus makes them continuous at $q=0$.

${ }^{15}$ K. Nomura and S. Takada, J. Phys. Soc. Jpn. 60, 389 (1991).

${ }^{16}$ In the case of Pauli matrices (spin-1/2), the spin operator $S^{3}$ and the center operator coincide.

${ }^{17}$ R. Haydock, in Solid State Physics: Advances in Research and Applications, edited by Editor(s) (Academic, New York, 1980), Vol. 35, p. 215; M. H. Lee, Phys. Rev. B 26, 2547 (1982); E. R. Gagliano and C. A. Balseiro, ibid. 38, 11766 (1988).

${ }^{18}$ J. Deisz, Phys. Rev. B 46, 2885 (1992). (iii) It can be shown that the trimer states (A4) are eigenstates of the Hamiltonian

$$
\begin{aligned}
H_{T}= & \sum_{n=1}^{N}\left(\mathcal{P}_{n, n+1}+\frac{1}{2} \mathcal{P}_{n, n+2}+\frac{1}{4} \mathcal{P}_{n, n+3}\right. \\
& \left.-\frac{1}{8} \mathcal{P}_{n, n+2} \mathcal{P}_{n+1, n+3}-\frac{1}{8} \mathcal{P}_{n+1, n+2} \mathcal{P}_{n, n+3}\right),
\end{aligned}
$$

with eigenvalues $-N / 2$. The permutation operator expressed in terms of spin-1 operators reads

$$
\mathcal{P}_{n, m}=\mathbf{S}_{n} \cdot \mathbf{S}_{m}+\left(\mathbf{S}_{n} \cdot \mathbf{S}_{m}\right)^{2}-1
$$

Finite- $N$ data indicate that the trimer eigenstates are, in fact, ground states.
${ }^{19}$ S. R. White and D. A. Huse, Phys. Rev. B 48, 3844 (1993).

${ }^{20}$ For this extrapolation we have used the BST algorithm, see R. Bulirsch and J. Stoer, Numer. Math. 6, 413 (1964); A. J. Guttmann, Phase Transition and Critical Phenomena, edited by C. Domb and J. L. Lebowitz (Academic, New York, 1989), Vol. 13 , p. 1 ..

${ }^{21}$ Whether the singlet two-magnon continuum observed in Fig. 2(c) really collapses into a number of $\propto N$ of degenerate excitations at $q=\pi$ cannot be decided on the basis of the recursion method. If the excitation at $\omega=\sqrt{10} J$ were degenerate, the recursion algorithm would pick out a particular direction in the subspace of these excitations such that $|\langle\lambda|A| G\rangle|^{2}=\left\langle G\left|A^{\dagger} A\right| G\right\rangle$ and $\left\langle\lambda^{\prime}|A| G\right\rangle=0$ for other (orthogonal) eigenvectors.

${ }^{22}$ Bound states with positive interaction energy are a common occurrence in quantum systems on the lattice where $H$ and $-H$ are equally well defined.

${ }^{23}$ Note of caution: States near the top or bottom of a continuum, which are more easily tracked than others, have transition rates which are likely to be affected by the singularity of the spectralweight distribution at the band edge in the limit $N \rightarrow \infty$.

${ }^{24}$ M. Karbach and G. Müller, Comput. Phys. 11, 36 (1997).

${ }^{25}$ The $\theta$ dependence of $S_{S S}(\pi)$ has a maximum at $\theta>-\pi / 4$ for finite $N$; as $N$ increases, the maximum grows and moves toward $\theta=-\pi / 4$.

${ }^{26}$ The $\theta$ dependence $S_{Z Z}(2 \pi / 3)$ has a maximum at $\theta<\pi / 4$ for finite $N$; as $N$ increases, the maximum grows and moves toward $\theta=\pi / 4$.

${ }^{27}$ J. Kurmann, H. Thomas, and G. Müller, Physica A 112, 235 (1982).

${ }^{28}$ E. Lieb, T. Schulz, and D. Mattis, Ann. Phys. (N.Y.) 16, 407 (1961).

${ }^{29}$ J. H. Taylor and G. Müller, Phys. Rev. B 28, 1529 (1983).

${ }^{30}$ The vectors $\left|\Phi_{k}^{D}\right\rangle, k=1,2$ are nonorthogonal for finite $N$, but they become orthogonal as $N \rightarrow \infty$. The same is true for the vectors $\left|\Phi_{k}^{T}\right\rangle, k=1,2,3$.

${ }^{31}$ I. Bose, S. Chatterjee, and C. K. Majumdar, Phys. Rev. B 29, 2741 (1984).

${ }^{32}$ C. K. Majumdar and D. K. Ghosh, J. Math. Phys. 10, 1388 (1969). 